\title{
Optimization of Combinational Logic Circuits Based on Compatible Gates *
}

\author{
Maurizio Damiani ${ }^{\dagger} \quad$ Jerry Chih-Yuan Yang Giovanni De Micheli
}

\author{
Center for Integrated Systems \\ Stanford University, Stanford CA 94305
}

\begin{abstract}
This paper presents a set of new techniques for the optimization of multiple-level combinational Boolean networks. Such techniques are based on a temporary transformation of the network into an internally unate one. We describe first a technique based upon the selection of appropriate multiple-output subnetworks (consisting of so-called compatible gates) whose local functions can be optimized simultaneously. We then generalize the method to larger subsets of unate gates. Because simultaneous optimization of local functions can take place, our methods are more powerful and general than Boolean optimization methods using don't cares, where only single-gate opti. mization can be performed. In addition, our methods represent a more efficient alternative to Boolean relations-based optimization procedures because the problem can be modeled by a unate covering problem instead of the more difficult binate covering problem. The method is implemented in program achilles and compares favorably to Sis.
\end{abstract}

\section{Introduction}

Logic synthesis has been traditionally divided into two-level and multiple-level synthesis. Two-level synthesis has been intensely researched from theoretical and engineering perspectives, and efficient algorithms for exact and approximate solutions are available [1]

Exact optimization algorithms for multiple-level logic networks have also been considered [2]. They are, however, generally impractical even for medium-sized networks. For this reason, many efficient approximation algorithms have been developed over the past decade. Such algorithms can be classified according to the algebraic/Boolean type of operations they perform. Algebraic techniques, such as factoring and kerneling, are described in [3].

As algebraic methods do not take full advantage of the properties of Boolean algebra, a spectrum of Boolean optimization techniques has been developed in parallel. Such techniques consist mainly of iteratively refining an initial network by identifying subnetworks to be optimized, deriving their associated degrees of freedom (expressed by so-called don't care conditions), and replacing such subnetworks by simpler, optimized ones.

The independent optimization of the local function of a network, called single-gate optimization, lies at one end of the spectrum. It has been shown $[4,5]$ that the degrees of freedom associated to a single gate can be represented by a don't care set. Once this set is obtained, two-level synthesis algorithms can be used to optimize the subnetwork [5].

The concurrent optimization of several local functions, called multiple-gate optimization, lies at the other end of the spectrum. Such methods have been shown to offer potentially better quality networks as compared to single-gate optimization because the degrees of freedom of multiple gates are used simultaneously. Heuristic approximations to multiple-gate optimization include the use of compatible don't cares [4] which allows us to extend don't care based optimization to multiple functions by restricting the don't care sets themselves. Although such methods are applicable to large networks, the restriction placed on don't care sets reduces the degrees of freedom and hence possibly the quality of the results. Exact methods for multiple-gate optimization, first analyzed in [6], have been shown to best exploit the degrees of freedom. Unfortunately these methods suffer from two major disadvantages. First, even for small subnetworks, the number of primes that have to be derived can be remarkably large; second, given the set of primes, it

*This research is sponsored by NSF and DEC under a PYI awand and by ARPA and NSF under contract MIP 8719546.

${ }^{\dagger}$ Now with the Dipartimento di Elettronica ed Informatica, Universitá di Padova, Via Gradenigo 6/A, Padova, Italy. entails the solution of an often complex binate covering problem, for which efficient algorithms are still the subject of investigation. As a result, the overall efficiency of the method is limited, and only relatively small networks can currently be handled.

The binate nature of the covering problem arises essentially from the arbitrariness of the subnetwork selected for optimization. In this paper, we develop alternative techniques for the optimization of multiple-output subnetworks. These techniques are based upon a temporary transformation of a network into an internally unate one, and on an accurate choice of the subnetworks to be optimized. The difficult binate covering step is avoided, and yet an optimization quality superior to don't care -based methods with comparable efficiency is achieved because multiple local functions can be optimized simultaneously. To this regard, first we introduce the notion of compatible set of gates as a subset of gates whose optimization can be solved exactly by classical two-level synthesis algorithms. We show that the simultaneous optimization of compatible gates allows us to reach optimal solutions not achievable by conventional don't care methods. We then leverage upon these results and present an algorithm for the optimization of more general subnetworks in an internally unate network. The algorithms have been implemented and tested on several benchmark circuits, and the results in terms of literal savings as well as CPU time are very promising.

\section{Terminology}

Let $\mathcal{B}$ denote the Boolean set $\{0,1\}$. A $k$-dimensional Boolean vector $\mathrm{x}=\left[x_{1}, \cdots, x_{k}\right]^{T}$ is an element of the set $\mathcal{B}^{k}$ (bold-facing is hereafter used to denote vector quantities. In particular, the symbol 1 denotes a vector whose components are all 1).

A $n_{i}$-input, $n_{0}$-output Boolean function $\mathbf{F}$ is a mapping $\mathbf{F}$ : $\mathcal{B}^{n_{2}} \rightarrow B^{n_{0}}$. A literal function, or literal, is the function expressed by a variable or its complement. A cube $c$ is the product of some literals. A gate refers to the local function that the output of the gate represents. A Boolean function can also be represented as a set using its minterms of the ON-set. For the rest of the paper, we interchangeably use set $(C, \supseteq)$ and function $(+, \cdot)$ notations to describe Boolean functions for notational convenience.

The cofactors (or residues) of a function $F$ with respect to a variable $x_{2}$ are the functions $\mathbf{F}_{x_{1}}=\mathbf{F}\left(x_{1}, \ldots, x_{2}=1, \ldots, x_{n}\right)$ and $\mathbf{F}_{x^{\prime}}=\mathbf{F}\left(x_{1}, \ldots, x_{1}=0, \ldots, x_{n}\right)$. The universal quantification or consensus of a function $F$ with respect to a variable $x_{a}$ is the function $\forall_{x_{i}} \mathbf{F}=\mathbf{F}_{x_{i}} \mathbf{F}_{x^{\prime}}$. A scalar function $F_{1}$ contains $F_{2}$ (denoted by $F_{1} \supseteq F_{2}$ ) if $F_{2}=1$ implies $F_{1}=1$. The containment relation holds for two vector functions if it holds component-wise.

A function $F$ is termed positive unate in $x_{i}$ if $F_{x_{1}} \supseteq F_{x_{i}^{i}}$, and negative unate if $F_{x_{1}} \subseteq F_{x^{\prime}}$. Otherwise the function is termed binate in $x_{i}$. A function $F$ positive unate in a variable $x_{1}$ can always be expressed without using the literal $x_{i}^{\prime}[7]$.

The desired terminal behavior of a combinational network is specified by two functions, $O N(x)$ and $D C(x)$, the latter in particular representing the input combinations that either do not occur or such that the value of some of the network outputs is regarded as irrelevant [5].

The functions $\mathrm{ON}$ and DC identify the set of possible terminal behaviors for the network: specifications are met by an implementation, realizing a function $F(x)$ if and only if $F(x)=O N(x)$ for every input $\mathbf{x}$ not in $\mathbf{D C}$.

Another, equivalent, description of the set of terminal behaviors is in terms of the functions $\mathbf{F}_{\min }=\mathbf{O N} \cdot \mathbf{D} \mathbf{C}^{\prime}$ and $\mathbf{F}_{\max }=\mathbf{O N}+$ DC. Specifications are met by $F$ if

$$
\mathbf{F}_{\min } \subseteq \mathbf{F} \subseteq \mathbf{F}_{\max }
$$

30th ACM/IEEE Design Automation Conference

Permission to copy without fee all or part of this material is granted provided that the copies are not made or distributed for direct commercial advantage, the ACM copyright notice and the title of the publication and its date appear, and notice is given that copying is by permission of the Association for Computing Machinery. To copy otherwise, or to republish, requires a fee and/or specific permission. (O)1993 ACM 0-89791-577-1/93/0006-0631 1.50 
We consider hereafter specifications directly in terms of a pair $\mathbf{F}_{\min }, \mathbf{F}_{\max }$

\subsection{Previous Work}

Most Boolean methods for multiple-level logic synthesis rely upon two-level synthesis engines. For this reason and in order to establish some essential terminology, we first review some basic concepts of two-level synthesis.

\section{Two-level Synthesis}

Consider the synthesis of a (single-output) network whose output $y$ is to satisfy $\mathrm{Eq}$. (1), imposing a realization of $y$ as a sum of cubes $c_{k}$ :

$$
F_{\min } \subseteq y=\sum_{k=1}^{N} c_{k} \subseteq F_{\max }
$$

The upper bound in Eq. (2) holds if and only if each cube $c_{k}$ satisfies the inequality

$$
c_{k} \subseteq F_{\max } \text {. }
$$

Any such cube is termed an implicant. An implicant is termed prime if no literal can be removed from it without violating the inequality (3). For the purpose of logic design, only prime implicants need be considered $[7,1]$. Each implicant $c_{k}$ has a cost $w_{k}$ associated to it, which depends on the technology under consideration. For example, in PLA minimization all implicants take the same area, and therefore have identical cost; in a multiple-level context, the number of literals can be taken as cost measure [3]. The cost of a sum of implicants is usually taken as the sum of the individual costs.

Once the list of primes has been built, a minimum-cost cover of $F_{\min }$ is determined by solving:

$$
\text { minimize : } \sum_{k=1}^{N} \alpha_{k} w_{k} ; \text { subject to: } F_{\min } \subseteq \sum_{k=1}^{N} \alpha_{k} c_{k}
$$

where the Boolean parameters $\alpha_{k}$ are used in this context to parameterize the search space: they are set to 1 if $c_{k}$ appears in the cover, and to 0 otherwise. The approach is extended easily to the synthesis of multiple-output circuits by defining multiple-output primes $[7,1]$. A multiple-output prime is a prime of the product of some components of $F_{\max }$. These components are termed the influence set of the prime.

Branch-and-bound methods can be used to solve exactly the problem. Engineering solutions have been thoroughly analyzed, for example, in [1], and have made two-level synthesis feasible for very large circuits.

Eq. (4) can be rewritten as

$$
\forall x_{1}, \ldots, x_{n}\left(\sum_{k=1}^{N} \alpha_{k} c_{k}(\mathbf{x})+F_{\min }^{\prime}(\mathbf{x})\right)=1 .
$$

The left-hand side of Eq. (5) represents a Boolean function $F_{a}$ of the parameters $\alpha_{i}$ only; the constraint equation (4) is therefore equivalent to

$$
F_{\alpha}=1 \text {. }
$$

The conversion of Eq. (4) into Eq. (6) is known in the literature as Petrick's method [7].

Two properties of two-level synthesis are worth remarking in the context of this paper. First, once the list of primes has been built, we are guaranteed that no solution will violate the upper bound in Eq. (1), so that only the lower bound needs to be considered (as explicited by Eq. (4)). Similarly, only the upper bound needs to be considered during the extraction of primes. Second, the effect of adding/removing a cube from a partial cover of $\mathbf{F}_{\min }$ is always predictable: that partial cover is increased/decreased. This property eases the problem of sifting the primes during the covering step, and it is reflected by the unateness of $F_{\alpha}$ : intuitively, by switching any parameter $\alpha$, from 0 to 1 , we cannot decrease the chances of satisfying Eq. (6). These are important attributes of the problem that need to be preserved in its generalizations.

\section{Don't care -based Multiple-level Optimization}

Two-level optimization is the basic engine in don't care -based multiple-level logic optimization, where it is used to iteratively optimize single-output gates in the network.

Consider a single-output subnetwork, with local output $y$, to be re-synthesized. The primary output $F$ of the overall network can be expressed in terms of the signal $y$ :

$$
\mathbf{F}=\mathbf{F}(\mathbf{x}, y)=y^{\prime} \mathbf{F}_{y^{\prime}}+y \mathbf{F}_{y}=\left(y \mathbf{1}+\mathbf{F}_{y^{\prime}}\right)\left(y^{\prime} \mathbf{1}+\mathbf{F}_{y}\right) \text {. }
$$

By replacing Eq. (7) in Eq. (1), it follows that $y$ must satisfy:

$$
\mathbf{F}_{\min } \subseteq y^{\prime} \mathbf{F}_{y^{\prime}}+y \mathbf{F}_{y} \subseteq \mathbf{F}_{\max } .
$$

A constraint on $y$ similar to Eq. (1) can be obtained from Eq (8) as follows. The upper bound in Eq. (8) holds if and only if $y^{\prime} \mathbf{F}_{y^{\prime}} \subseteq \mathbf{F}_{\max }$ and $y \mathbf{F}_{\mathbf{y}} \subseteq \mathbf{F}_{\max }$, i.e.

$$
y^{\prime} \subseteq \mathbf{F}_{\max }+\mathbf{F}_{y^{\prime}}^{\prime} ; \quad y \subseteq \mathbf{F}_{\max }+\mathbf{F}_{y}^{\prime} \text {. }
$$

Eq. (9) can be rewritten as

$$
\mathbf{F}_{\max }^{\prime} \mathbf{F}_{y^{\prime}} \subseteq y \mathbf{1} \subseteq \mathbf{F}_{\max }+\mathbf{F}_{y}^{\prime} .
$$

Similarly, the lower bound holds if and only if $\mathbf{F}_{y^{\prime}}+y 1 \supseteq \mathbf{F}_{\min }$ and $\mathbf{F}_{y}+y^{\prime} 1 \supseteq \mathbf{F}_{\min }$, i.e.

$$
\mathbf{F}_{\min } \mathbf{F}_{y^{\prime}} \subseteq y \mathbf{1} \subseteq \mathbf{F}_{\min }^{\prime}+\mathbf{F}_{y}
$$

Eq. (10) and (11) can be merged together, to obtain:

$$
\mathbf{F}_{\min } \mathbf{F}_{y^{\prime}}^{\prime}+\mathbf{F}_{\max }^{\prime} \mathbf{F}_{y^{\prime}} \subseteq y \mathbf{1} \subseteq\left(\mathbf{F}_{\max }+\mathbf{F}_{y}^{\prime}\right)\left(\mathbf{F}_{\min }^{\prime}+\mathbf{F}_{y}\right) \text {. }
$$

Eq. (12) represents the exact degrees of freedom available in the synthesis of the signal $y$, and is formally identical to Eq. (1): the value of $y$ is undetermined corresponding to those points for which the lower bound differs from the upper bound. Such points are the local don't cares for $y$, and are denoted by $\mathbf{D C}_{y}(\mathbf{x})$. Once the bounds (or, equivalently, the don't cares ) for $y$ are computed, ordinary two-level synthesis algorithms can be applied. ${ }^{\prime}$

\section{Boolean Relations-based Multiple-level Optimization}

Don't care -based methods allow the optimization of only one single-output subnetwork at a time. It has been shown in [6] that this strategy may potentially produce lower-quality results with respect to a more general approach attempting the simultaneous optimization of multiple-output subnetworks.

Let $\mathbf{y}=\left[y_{1}, y_{2}, \cdots, y_{m}\right]$ denote the outputs of a subnetwork, to be re-synthesized, and let $\mathbf{F}(\mathbf{x}, \mathbf{y})$ denote the network outputs, expressed in terms of the variables $y_{1}$. From equation(1), the functional constraints on $y$ are expressed by

$$
\mathbf{F}_{\min } \subseteq \mathbf{F}(\mathbf{x}, \mathbf{y}) \subseteq \mathbf{F}_{\max } .
$$

An equation like Eq. (13) describes a Boolean Relation ${ }^{2}$. The synthesis problem consists of finding a minimum-cost realization of $y_{1}, \ldots, y_{m}$ such that Eq. (13) holds. An exact solution algorithm, targeting two-level realizations, is presented in [6].

The difficulty with solutions to Boolean relation is twofold: First, when trying to express Eq. (13) in a form similar to Eq. (12), the isolation of a particular $y_{2}$ results in dependence of $y$, in the upper and lower bounds of the expression. This makes simultaneous optimization of $y_{1} \ldots y_{m}$ difficult. Second, Eq. (13) requires a solution to the binate covering problem in the covering step. Fast binate covering solvers are the subject of ongoing research [8]. Nevertheless, the binate nature of the covering step reflects an intrinsic complexity which is not found in the unate case. In particular, since $\mathbf{F}$ is in general binate with respect to $y$, the effect of adding / removing a prime to a partial solution is no longer trivially predictable and both bounds in Eq. (13) may be violated by the addition of a single cube. As a consequence, branch-and-bound solvers may (and usually do) undergo many more backtracks than with a unate problem of comparable size, resulting in a substantially increased CPU time.

${ }^{1}$ In practice, $y$ is re-synthesized by taking advantage also of the other internal signals available in the network. Implicants and primes are in this context expressed in terms of primary inputs and other network variables.

${ }^{2}$ An alternative formulation of a Boolean Relation is by means of a characteristic equation: $R(x, y)=1$, where $R$ is a Boolean function. It could be shown that the two formulations are equivalent. 


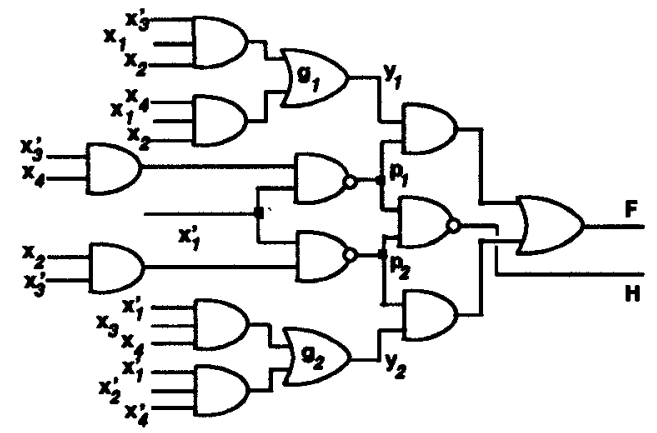

Figure 1: Gates $g_{1}$ and $g_{2}$ are compatible.

\section{Compatible Gates}

The analysis of Boolean relations points out that binate problems arise because of the generally binate dependence of $F$ on the variables $y_{i}$. We introduce the notion of compatible gates in order to perform multiple-gate optimization while avoiding the binate covering problem. In the rest of the paper, given a network output expression $\mathbf{F}(x, y), x$ is the set of input variables and $y$ is the set of gate outputs to be optimized.

Definition 1 A subset of gates $\mathcal{S}=\left\{g_{1}, \ldots, g_{k}\right\}$ in a Boolean network with outputs $y_{1} \ldots y_{m}$ is said to be compatible if the function F can be expressed in terms of the network outputs as

$$
\mathbf{F}_{k}=\sum_{j=1}^{m} y_{j} \mathbf{p}_{j}+\mathbf{q} ;
$$

modulo a phase change in the variables $y_{j}$ or F. In Eq. (14), the functions $p_{j}=p_{j}\left(x_{1}, \ldots, x_{n}\right)$ and $q=q\left(x_{1}, \ldots, x_{n}\right)$ do not depend on $y_{1}, \ldots, y_{m}$.

Compatible gates allow optimization of multiple gates without having to solve the binate covering problem. Intuitively, compatible gates are selected such that their optimization can only affect the outputs in a monotonic or unate way, and thereby forcing the covering problem to be unate.

Example 1 Consider the two-output circuit in Figure 1. Gates $g_{1}$ and $g_{2}$ are compatible because $F$ and $H$ can be written as

$$
\begin{gathered}
F=\left(x_{1}+x_{3}+x_{4}^{\prime}\right) y_{1}+\left(x_{1}+x_{2}^{\prime}+x_{3}\right) y_{2}, \\
H=0 y_{1}+0 y_{2}+\left(\left(x_{1}+x_{3}+x_{4}^{\prime}\right)\left(x_{1}+x_{2}^{\prime}+x_{3}\right)\right)^{\prime} ;
\end{gathered}
$$

The compatibility of a set $\mathcal{S}$ of gates is a Boolean property. In order to ascertain it, one would have to verify that all network outputs can indeed be expressed as in Definition (3). This task is potentially very CPU-intensive. In Appendix A, we present algorithms for constructing subsets of compatible gates from the network topology only.

\subsection{Optimizing Compatible Gates}

The functional constraints for a set of compatible gates can be obtained by replacing Eq. (14) into Eq. (13). From Eq. (14) we obtain:

$$
\mathbf{F}_{\min } \subseteq \sum_{j=1}^{m} y_{j} \mathbf{p}_{j}+\mathbf{q} \subseteq \mathbf{F}_{\max }
$$

Eq. (15) can be solved using steps similar to that of two-level optimization. In particular, the optimization steps consist of implicant extraction and the covering steps.

\section{Implicant Extraction}

Assuming that $q \subseteq F_{\max }$, the upper bound of Eq. (15) holds if and only if for each product $y_{j} \mathbf{p}_{j}$ the inequality

$$
\boldsymbol{y}_{j} \mathbf{p}_{j} \subseteq \mathbf{F}_{\max }
$$

is verified, i.e. if and only if

$$
y_{j} \mathbf{1} \subseteq \mathbf{F}_{\max }+\mathbf{p}_{j}^{\prime} ; j=1, \ldots, m .
$$

\begin{tabular}{|c|c|c|}
\hline & Primes & Influence sets \\
\hline$c_{1}$ & $x_{1}^{\prime} x_{2}^{\prime} x_{3}$ & $y_{1}, y_{2}$ \\
$c_{2}$ & $x^{\prime} x_{2}^{\prime} x_{4}^{\prime}$ & $y_{1}, y_{2}$ \\
$c_{3}$ & $x_{1}^{\prime} x_{3} x_{4}$ & $y_{1}, y_{2}$ \\
$c_{4}$ & $x_{2} x_{4}$ & $y_{1}, y_{2}$ \\
$c_{5}$ & $x_{1} x_{2} x_{3}^{\prime}$ & $y_{1}, y_{2}$ \\
$c_{6}$ & $x_{2} x_{3}^{\prime}$ & $y_{2}$ \\
$c_{7}$ & $x_{1}^{\prime} x_{3}^{\prime} x_{4}^{\prime}$ & $y_{2}$ \\
$c_{8}$ & $x_{1}^{\prime} x_{2}^{\prime}$ & $y_{1}$ \\
$c_{9}$ & $x_{1}^{\prime} x_{4}$ & $y_{1}$ \\
\hline
\end{tabular}

Table 1: Multiple-output primes for Example (2).
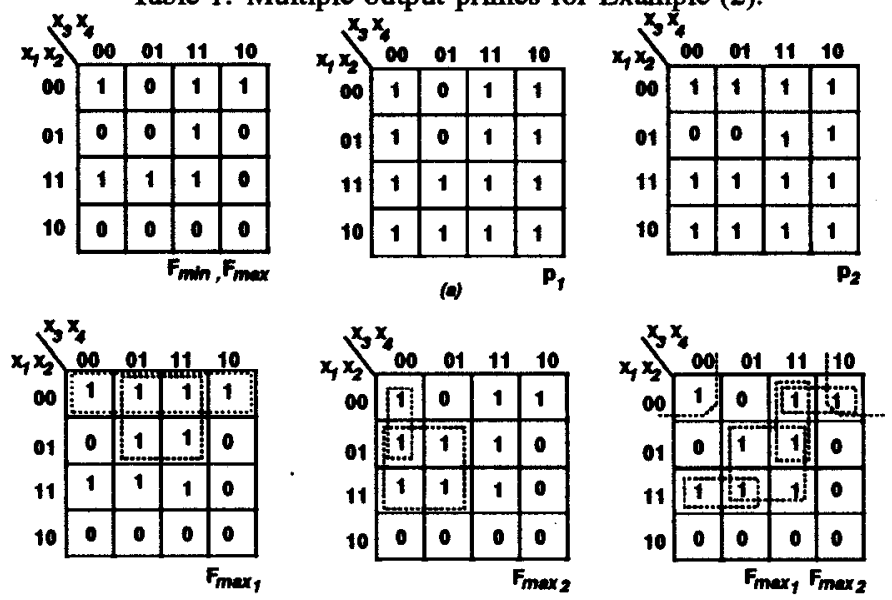

(b)

Figure 2: (a): Maps of $F_{\min }, F_{\max }, p_{1}, p_{2}$. (b) Maps of $F_{\max , 1}, F_{\max , 2}$ and of the product $F_{\max , 1} F_{\max , 2}$. Primes of $y_{1}$ and $y_{2}$ are shown in the maps of $F_{\max , 1}$ and $F_{\max , 2}$, respectively. The map of $F_{\max , 1} F_{\max , 2}$ shows the primes common to $y_{1}$ and $y_{2}$.

or, equivalently,

$$
y_{j} \subseteq F_{\max , j} ; j=1, \ldots, m
$$

where $F_{\max , j}$ is the product of all the components of $\mathbf{F}_{\max }+\mathbf{p}_{j}^{\prime}$. A cube $c$ can thus appear in a two-level expression of $y_{j}$ if and only if $c \subseteq F_{\max , j}$. As this constraint is identical to Eq. (3), the primeextraction strategies $[7,1]$ of ordinary two-level synthesis can be used.

Example 2 Consider the optimization problem for gates $g_{1}$ and $g_{2}$ in Fig. (1).

From Example (1),

$$
\begin{aligned}
& p_{1}=\left(x_{1}+x_{3}+x_{4}^{\prime}\right)^{\prime} ; \\
& p_{2}=\left(x_{1}+x_{2}^{\prime}+x_{3}\right)^{\prime} .
\end{aligned}
$$

We assume no external don't care set. Consequently, $F_{\min }=$ $F_{\max }=x_{1} x_{2} x_{3}^{\prime}+x_{2} x_{3} x_{4}+x_{1}^{\prime} x_{2}^{\prime}\left(x_{3}+x_{4}^{\prime}\right)$. The Karnaugh maps of $F_{\min }$ and $F_{\max }$ are shown in Fig. $(2 a)$, along with those of $p_{1}$ and $p_{2}$. Fig. (2b) shows the maps of $F_{\max , 1}=F_{\max }+p_{1}^{\prime}$ and $F_{\max , 2}=F_{\max }+p_{2}^{\prime}$, used for the extraction of the primes of $y_{1}$ and $y_{2}$, respectively. The list of all multiple-output primes is given in Table (1). Note that primes 1 through 5 can be used by both $y_{1}$ and $y_{2}$.

\section{Covering Step}

Let $N$ indicate the number of primes. For example, in the problem of Example (2), $N=9$. We then impose a sum-of-products representation associated with each variable $y_{j}$ :

$$
y_{j}=\sum_{k=1}^{N} \alpha_{j k} c_{k}
$$

with the only restriction that $\alpha_{j k}=0$ if $y_{j}$ is not in the influence set of $c_{k}$. Since the upper bound of Eq. (15) is now satisfied by construction (i.e. by implicant computation), the minimization of 

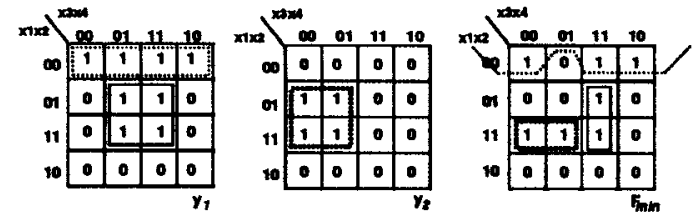

Figure 3: A minimum-cost solution for the covering of $F_{\min }$.

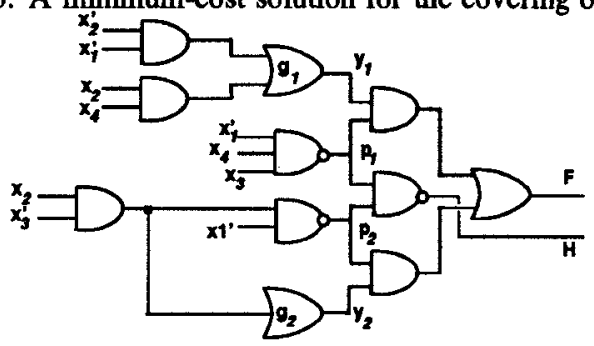

Figure 4: Network resulting from the simultaneous optimization of compatible gates $g_{1}$ and $g_{2}$.

$y_{1}, \ldots, y_{m}$ can be formulated as a minimum-cost covering problem

$$
\mathbf{F}_{\min } \subseteq \mathbf{q}+\sum_{j=1}^{m} \sum_{k=1}^{N} \alpha_{j k} c_{k} \mathbf{p}_{j}
$$

whose similarity with $\mathrm{Eq}$. (4) is evident, the products $c_{j k} \mathbf{p}_{j}$ now playing the role of the primes of two-level synthesis.

Example 3 In the optimization problem of Example (2), we are to solve the covering problem

$$
F_{\min } \subseteq p_{1} y_{1}+p_{2} y_{2} .
$$

Using the set of primes found in Example (2), $y_{1}$ and $y_{2}$ are expressed by

$$
\begin{array}{r}
y_{1}=\alpha_{1,1} c_{1}+\alpha_{1,2} c_{2}+\alpha_{1,3} c_{3}+\alpha_{1,4} c_{4}+\alpha_{1,5} c_{5}+ \\
\alpha_{1,8} c_{8}+\alpha_{1,9} c_{9} \\
y_{2}=\alpha_{2,1} c_{1}+\alpha_{2,2} c_{2}+\alpha_{2,3} c_{3}+\alpha_{2,4} c_{4}+\alpha_{2,5} c_{5}+ \\
\alpha_{2,6} c_{6}+\alpha_{2,7} c_{7}
\end{array}
$$

The optimum solution has cost 6 and is given by $y_{1}=x_{1}^{\prime} x_{2}^{\prime}+$ $x_{2} x_{4} ; y_{2}=x_{2} x_{3}^{\prime}$, corresponding to the assignments

$$
\begin{aligned}
& \alpha_{1,1}=\alpha_{1,2}=\alpha_{1,3}=\alpha_{1,5}=\alpha_{1,9}=0 ; \quad \alpha_{1,4}=\alpha_{1,8}=1 ; \\
& \alpha_{2,1}=\alpha_{2,2}=\alpha_{2,3}=\alpha_{2,4}=\alpha_{2,5}=\alpha_{2,7}=0 ; \quad \alpha_{2,6}=1 .
\end{aligned}
$$

The initial cost, in terms of literals, was 12. The solution corresponds to the cover shown in Fig. (3), and resulting in the circuit of Fig. (4).

It is worth contrasting, in the above example, the role of $y_{1}$ and $y_{2}$ in covering $F_{\min }$. Before optimization, $p_{1} y_{1}$ covered the minterms $x_{1} x_{2} x_{3}^{\prime} x_{4}^{\prime}, x_{1} x_{2} x_{3}^{\prime} x_{4}, x_{1} x_{2} x_{3} x_{4}$ of $F_{\min }$, while $p_{2} y_{2}$ covered $x_{1}^{\prime} x_{2}^{\prime} x_{3}^{\prime} x_{4}^{\prime}, x_{1}^{\prime} x_{2}^{\prime} x_{3} x_{4}^{\prime}, x_{1}^{\prime} x_{2} x_{3} x_{4}, x_{1}^{\prime} x_{2}^{\prime} x_{3} x_{4}$. After optimization, $y_{1}$ and $y_{2}$ essentially "switched role" in the cover: $p_{2} y_{2}$ is now used for covering $x_{1} x_{2} x_{3}^{\prime} x_{4}^{\prime}, x_{1} x_{2} x_{3}^{\prime} x_{4}$, while $p_{1} y_{1}$ covers all other minterms.

In the general case, the possibility for any of $y_{1}, \ldots, y_{m}$ to cover a minterm of $F_{\min }$ is evident from Eq. (15). Standard single-gate optimization methods based on don't care [5] regard the optimization of each gate $g_{1}, \ldots, g_{m}$ as separate problems, and therefore this degree of freedom is not used. For example, in the circuit of Fig. (1), the optimization of $g_{1}$ is distinct from that of $g_{2}$. The don't care conditions associated to (say) $y_{1}$ are those minterms for which either $p_{1}=0$ or such that $p_{2} y_{2}=1$, and are shown in the map of Fig. (5), along with the initial cover. It can immediately be verified that $y_{1}$ can only be optimized into $x_{1} x_{2} x_{3}^{\prime}+x_{2} x_{4}$, saving only one literal.

The don't cares for $y_{2}$ are also shown in Fig. (5). No optimization is possible in this case. Note also that the optimization result is (in this particular example) independent from the order in which $g_{1}$ and $g_{2}$ are optimized. Unlike the compatible gates case, it is impossible for the covers of $y_{1}$ and $y_{2}$ to "switch" role in covering $F_{\min }$.
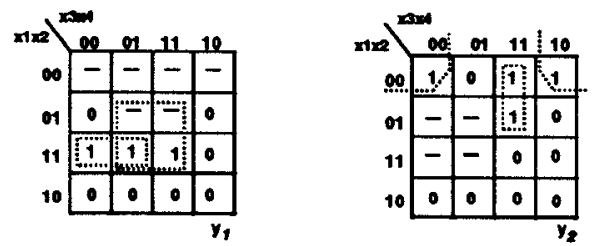

Figure 5: Don't care conditions associated with $y_{1}$ and $y_{2}$ : only 1 literal can be removed.

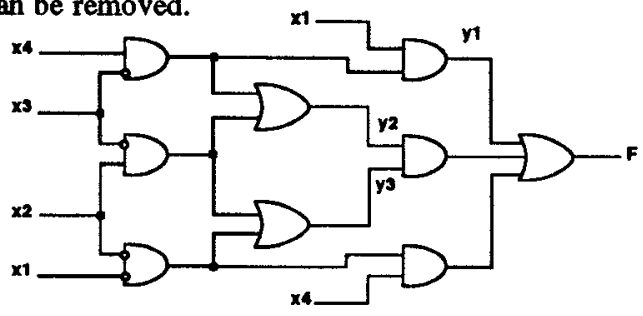

Figure 6: Network for Example (4).

\section{Unate Optimization}

In the previous section we showed that in the case of compatible gates, the functional constraints expressed by Eq. (13) can be reduced to Eqs. (17) and (19), which could be solved by a two-step procedure similar to that of two-level optimization. We now generalize the compatible gates results to the optimization of arbitrary subsets $\mathcal{S}$ of unate gates.

\subsection{Optimizing Unate Subsets}

Assume, for the sake of simplicity, that $F$ is positive unate with respect to $y_{1}, \ldots, y_{m}$. We can perform optimization on the subset of unate gates in a style that is totally analogous to compatible gates by dividing it into implicant extraction and covering steps.

Implicant Extraction

In this step, for each $y_{i}$ to be optimized, a set of maximal functions is extracted. In particular, the maximal functions of each each $y_{i}$ can be expressed as Eq. (20), which is similar to Eq. (17).

$$
y_{i} \subseteq F_{\max , j} ; j=1, \ldots, m \text {; }
$$

From Eq. (20), appropriate implicants can then be extracted.

Intuitively, the maximal functions are the largest functions that can be used while satisfying the bound $\mathbf{F} \subseteq \mathbf{F}_{\max }$. Therefore, they represent the upper bounds on $y_{i}$. We introduce the following definition:

\section{Definition 2 A set of functions}

$$
\left\{F_{\max , 1}(\mathbf{x}), F_{\max , 2}(\mathbf{x}), \ldots, F_{\max , m}(\mathbf{x})\right\}
$$

is said to be maximal if

$$
\mathbf{F}\left(\mathbf{x}, F_{\max , 1}(\mathbf{x}), F_{\max , 2}(\mathbf{x}), \ldots, F_{\max , m}(\mathbf{x})\right) \subseteq \mathbf{F}_{\max }
$$

and the inequality (21) is violated only when any $F_{\max }$ is replaced by a larger function $\supset F_{\max _{j}}$.

By substituting the maximal functions for each $y_{i}, \mathrm{Eq}$. (13) can be reduced to the following:

$$
\mathbf{F}_{\min } \subseteq \mathbf{F}(\mathbf{x}, \mathbf{y})
$$

The remaining task is to find a minimum-cost covering solution for Eq. (22). The following theorem, whose proof is in [9], provides means for finding a set of maximal functions. It also shows that computing such functions has complexity comparable with computing ordinary don't care sets.

Theorem 4.1 Maximal functions can be obtained as

$$
F_{\max , j}=f_{j}+D C_{j}
$$

where $D C_{j}$ repnesents the don't care set associated with $y_{j}$, as. suming that $y_{k}=F_{\max , k}, k=1, \ldots, j-1$ and $y_{k}=f_{k} ; k=$ $j+1, \ldots, m$.

This theorem states that the maximal function for vertex $i$ depends on the maximal functions already calculated $(j<i)$. This means that unlike the case of compatible gates, maximal function for a given vertex is not unique. 
Example 4 For the network of Fig. (6), assuming no external don't care conditions, we find the maximal functions for $y_{1}, y_{2}$, and y3. The $D C_{y_{1}}$ terms correspond to the observability don't care at $y_{i}$, computed using the $F_{\max }$ of the previous gates.

$$
y_{1}=x_{1} x_{3}^{\prime} x_{4} ; \quad y_{2}=x_{3}^{\prime}\left(x_{4}+x_{2}\right) ; \quad y_{3}=x_{3}^{\prime} x_{2}+x_{1}^{\prime} x_{2}^{\prime}
$$

Maximal functions derived by Theorem (4.1) are :

$$
\begin{aligned}
& F_{\max , 1}=x_{1} x_{3}^{\prime} x_{4}+D C_{y_{1}}=x_{3}^{\prime} x_{4}+\left(x_{3}^{\prime}+x_{4}\right) x_{1}^{\prime} x_{2}^{\prime} \\
& \begin{aligned}
F_{\max , 2} & =x_{3}^{\prime}\left(x_{4}+x_{2}\right)+D C_{y_{2}}\left(y_{1}=F_{\max , 1}\right) \\
& =x_{4}+x_{3}^{\prime} x_{2}^{\prime}+x_{1} x_{2}^{\prime}+x_{3} x_{2}
\end{aligned} \\
& \begin{aligned}
F_{\max , 3}= & x_{3}^{\prime} x_{2}^{\prime}+x_{1}^{\prime} x_{2}+D C_{y_{3}}\left(y_{1}=F_{\max , 1}, y_{2}=F_{\max , 2}\right) \\
= & x_{3}^{\prime} x_{2}+x_{1}^{\prime} x_{2}^{\prime}+x_{4} x_{3}^{\prime}
\end{aligned}
\end{aligned}
$$

\section{Covering Step}

Eq. (20) allows us to find a set of multiple-output primes for $y_{1}, \ldots, y_{m}$. The covering step then consists of finding a minimumcost sum such that Eq. (22) holds.

We now present a reduction for transforming the covering step to the one presented for compatible gates. We first illustrate the reduction by means of an example.

Example 5 In Fig. (6), consider the combination of inputs $\mathrm{x}$ resulting in $F_{\min }(\mathbf{x})=1$. To each such combination we can associate the set of values of $y_{1}, y_{2}, y_{3}$ such that $F(\mathbf{x}, \mathbf{y})=1$. For instance, for the entry $x_{1} x_{2} x_{3} x_{4}=1001$, it must be $F_{x_{1} x_{2}^{\prime} x_{3}^{\prime} x_{4}}(y)=$ $y_{1}+y_{2} y_{3}=1$. Let us now denote with $G(y)$ the left-hand side of this constraint, i.e. $G(\mathbf{y})=y_{1}+y_{2} y_{3}$. Notice that $G(\mathbf{y})$ is unate in each $y$, and changes depending on the combination of values currently selected for $x_{1}, x_{2}, x_{3}, x_{4}$.

Any constraint $G(\mathbf{y})=1$ can be represented in a canonical form:

$$
\begin{array}{r}
G(\mathbf{y})=\left(G_{y_{1}^{\prime} y_{2}^{\prime} y_{3}^{\prime}}+y_{1}+y_{2}+y_{3}\right)\left(G_{y_{1}^{\prime} y_{2}^{\prime} y_{3}}+y_{1}+y_{2}\right) \\
\ldots\left(G_{y_{1} y_{2} y_{3}^{\prime}}+y_{3}\right) G_{y_{1} y_{2} y_{3}}=1
\end{array}
$$

which, in tum, is equivalent to the 8 constraints

$$
\begin{aligned}
& G_{y_{1}^{\prime} y_{2}^{\prime} y_{3}^{\prime}} y_{1}+y_{2}+y_{3}=1 \\
& G_{y_{1}^{\prime} y_{2}^{\prime} y_{3}}+y_{2}+y_{3}=1 \\
& \dddot{G}_{y_{1} y_{2} y_{3}^{\prime}}+\quad y_{3}=1 \\
& G_{y_{1} y_{2} y_{3}}=1
\end{aligned}
$$

By introducing an auxiliary variable $z_{1}$ for each $y_{i}$, we can rewrite Eq. (24) as:

$$
G(\mathrm{z})+z_{1}^{\prime} y_{1}+z_{2}^{\prime} y_{2}+z_{3}^{\prime} y_{3}=1 \quad \forall z_{1}, z_{2}, z_{3} .
$$

or, equivalently,

$$
G^{\prime}(\mathbf{z}) \subseteq z_{1}^{\prime} y_{1}+z_{2}^{\prime} y_{2}+z_{3}^{\prime} y_{3} \text {. }
$$

In this particular example, we get

$$
\left(z_{1}+z_{2} z_{3}\right)^{\prime} \subseteq z_{1}^{\prime} y_{1}+z_{2}^{\prime} y_{2}+z_{3}^{\prime} y_{3} \text {. }
$$

More generally, corresponding to each combination $x$ such that $F_{\min }(\mathbf{x})=1$, the constraint $F(\mathbf{x}, y)=1$ can be re-expressed as

$$
F(\mathbf{x}, \mathbf{z})+z_{1}^{\prime} y_{1}+z_{2}^{\prime} y_{2}+\ldots+z_{m}^{\prime} y_{m}=1 \text {. }
$$

The transformation shown in Example (5) is formalized by the following theorem (whose proof can be found in [9]):

Theorem 4.2 Let $z=\left[z_{1}, \ldots, z_{m}\right]$ denote $m$ auxiliary Boolean variables. $E q$. (22) holds if and only if

$$
\mathbf{F}_{\min } \subseteq \mathbf{F}(\mathbf{x}, \mathbf{z})+\sum_{j=1}^{m} y_{j}\left(z_{j}^{\prime} \mathbf{1}\right)
$$

Eq. (25) has the same format of Eq. (15), with $q$ and $p$, being replaced by $F(x, z)$ and $z_{j}^{\prime} 1$, respectively. Theorem (4.2) thus allows us to reduce the covering step to the one used for compatible gates. Theorems (4.1) and (4.2) show that the algorithms presented in Section 3 can be used to optimize arbitrary sets of gates with the same parity, without being restricted to sets of compatible gates only.

\section{Implementation and Results}

The implementation of the algorithms presented in Sections 3 and 4 is as follows. The original networks are first transformed into a unate, NOR-only description. All internal functions are represented using BDDs [10]. For each unoptimized gate $g_{i}$, the following heuristic is used. First, we try to find a set of comptible gates for $g_{2}$, called $\mathcal{S}_{c}$. In the case where not enough compatible gates can be found, we find a set of gates that are unate with respect to $g_{i}$, called $\mathcal{S}_{a}$.

In the case where $\mathcal{S}_{c}$ is optimized, we use Eq. (14) to extract the functions $\mathbf{p}_{j}$ and $\mathbf{q}$. In particular, $\mathbf{q}$ is extracted by simulating the network with outputs $y_{3}$ stuck-at 0 . The functions $\mathbf{p}_{j}$ are then extracted by simulating the network with $y$, stuck-at 1 , with $y_{i} ; i \neq$ $j$ stuck-at 0.

In the case of optimizing arbitrary unate network $\mathcal{S}_{a}$, Theorem (4.1) is used to determine the maximal functions for each $y_{j}$. Note that optimizing $\mathcal{S}_{c}$ is preferable because for a set of $m$ compatible gates, $m+1$ simulations are needed to obtain all the required don't cares. For $\mathcal{S}_{a}$, two simulations (with $y$, stuck-at-0 and stuck-at-1) are required for the extraction of the don't care set of each variable $y_{j}$, resulting in a total of $2 m$ simulations.

A set of primes for the gate outputs is then constructed. Because of the large possible set of primes, we limit our prime selection to single-literal primes only. The BDD of $F(x, z)$ is then built, and the covering problem solved. Networks are then iteratively optimized until no improvement occurs, and eventually folded back to a binate form. The algorithms presented in this paper were implemented in C program called achilles, and tested against a set of MCNC synthesis benchmarks.

Table (2) provides a comparison of achilles with Sis using script.rugged. The column Initial Stat. lists the network statistics before optimization, where Int. is number of internal interconnections and gates is the gate count. The column Interconn. shows number of interconnections after optimization. The gates column compares final gate counts. Literal column shows the final literals in factored form. The results in the table show that achilles performs better than Sis for all figures of merits. In particular, achilles does $11 \%$ better than Sis in factored literals.

Note that script.rugged was chosen because it is the most robust script of the Sis script suite, and it matches closely to our type of optimization. Our objective was to compare optimization results based only on Boolean operations, namely compatible gates versus don't cares. The script.rugged calls full_simplify[11], which computes observability don't cares to optimize the network.

The table shows that the achilles runtimes are competitive with that of SIS. In this first implementation, we are more interested in the quality of the optimization than the efficiency of the algorithms, therefore an exact covering solver is used. We can improve the runtime in the future by substituting a faster heuristic or approximate solvers (such as used in ESPRESSO [1]).

\section{Conclusion}

In this paper we presented a comparative analysis of approaches to multi-level logic optimization, and presented new algorithms for simultaneous multiple-gate optimization. The algorithms are based on the notion of compatible gates. We identify the main advantage of the present approach over previous solutions in its capability of exact minimization of suitable multiple-output networks, by means of traditional two-level optimization algorithms. Preliminary experimental results show an improvement of $11 \%$ over existing methods.

\section{Appendix A Finding Compatible Gates}

In this section, we describe an algorithm for finding compatible gates based on network topology. In this analysis, we make the assumption that the network is transformed into its equivalent NORonly form. In this case, the parity of a path is simply the parity of the path length.

In defining Equation (14) for compatible gates, it is evident that the dependency of $F$ on $y_{1}, \ldots, y_{m}$ must be unate. In order to increase the chances of finding sets of compatible gates, it is thus convenient to transform a network into an internally unate one. This 


\begin{tabular}{|c|c|c|c|c|c|c|c|c|c|c|}
\hline \multirow[b]{2}{*}{ Circuit } & \multicolumn{2}{|c|}{ Tnitial Stat. } & \multicolumn{2}{|c|}{ Interconn. } & \multicolumn{2}{|c|}{ Literals(fac) } & \multicolumn{2}{|c|}{ Gates } & \multicolumn{2}{|c|}{ CPU time } \\
\hline & Int. & Gates & Achilles & SIS & Achilles & SIS & Achilles & SIS & Achilles & SIS \\
\hline cm85a & 108 & 63 & 67 & 77 & 42 & 46 & 31 & 34 & 1.5 & 1.2 \\
\hline $\operatorname{cm} 162 a$ & 113 & 60 & 99 & 102 & 47 & 49 & 41 & 52 & 1.8 & 1.3 \\
\hline pm1 & 130 & 60 & 67 & 78 & 47 & 52 & 31 & 36 & 1.6 & 1.3 \\
\hline 9symml & 375 & 152 & 288 & 325 & 163 & 186 & 88 & 101 & 108.4 & 64.2 \\
\hline alu2 & 924 & 262 & 366 & 570 & 303 & 362 & 215 & 231 & 309.7 & 403.0 \\
\hline alu4 & 1682 & 521 & 902 & 1128 & 612 & 703 & 420 & 487 & 1612.6 & 1718.5 \\
\hline apex6 & 1141 & 745 & 1009 & 1315 & 687 & 743 & 589 & 639 & 115.1 & 30.3 \\
\hline C499 & 945 & 530 & 913 & 945 & 505 & 552 & 498 & 530 & 202.1 & 133.6 \\
\hline $\mathrm{C} 880$ & 797 & 458 & 643 & 731 & 355 & 409 & 295 & 342 & 340.6 & 30.7 \\
\hline C1908 & 936 & 489 & 828 & 891 & 518 & 542 & 445 & 482 & 422.1 & 138.8 \\
\hline
\end{tabular}

Table 2: Optimization Results. Runtimes are in seconds on DEC5000/240.

is done by duplicating those gates whose fanouts contain reconvergent paths with different inversion parity. The resulting network is therefore at most twice the size of the original one. In practice, the increase is smaller.

Definition 3 A network is termed unate with respect to a gate $\mathrm{g}$ if all reconvergent paths from $\mathbf{g}$ have the same parity of inversions. A network is internally unate if it is unate with respect to each of its gates. All paths from $g$ to a primary output $z_{i}$ in an internally unate network has parity $\pi_{i}$, which is defined to be the parity of $g$ with respect to $z_{i}$

Theorem (3.1) below provides a sufficient conditions for a set $\mathcal{S}$ of gates to be compatible. Without loss of generality, the theorem is stated in terms of networks with one primary output. The following auxiliary definitions are required:

Definition 4 The fanout gate set and fanout edge set of a gate $g$, indicated by $F O(g)$ and $F O E(g)$, respectively, are the set of gates and interconnections contained in at least one path from $g$ to the primary output.

The fanout gate set and fanout edge set of a set of gates $\mathcal{S}=$ $\left\{g_{1}, \ldots, g_{k}\right\}$, indicated by $F O(\mathcal{S})$ and $F O E(\mathcal{S})$, respectively, are:

$$
F O(S)=\bigcup_{i=1}^{|S|} F O\left(g_{i}\right) ; \quad F O E(S)=\bigcup_{i=1}^{|S|} F O E\left(g_{i}\right) ;
$$

Theorem A.1 In a NOR-only network, let $\mathcal{S}=\left\{g_{1}, \ldots, g_{m}\right\}$ be $a$ set of gates of parity $\pi$, and not in each others' fanout. Let $y_{1}, \ldots, y_{m}$ denote the respective outputs. The following propositions hold:

1) if each gate in $F O(\mathcal{S})$ with parity $\pi$ has at most one input interconnection in $F O E(\mathcal{S})$, then the primary outputs can be expressed as in Eq. (14) for some suitable functions $p_{j}$ and $q$;

2) if each gate in $F O(S)$ with parity $\pi^{\prime}$ has at most one input in $F O E(S)$, then it can be shown that the output can be expressed as in Eq. (14).

The proof can be found in [9].

Theorem (A.1) also provides a technique for constructing a set of compatible gates directly from the network topology, starting from a "seed" gate $g$ and a parameter ( $r u l e$ ) that specifies the desired criterion of Theorem (A.1) (either 1 or 2) to be checked during the construction. The algorithm is as follows:

$$
\begin{aligned}
& \text { COMPATIBLES(g, rule }) \\
& \text { label_fanout }(g, F O) ; \\
& \mathcal{S}=\{g\} \\
& \text { for(i }=0 ; i \leq N G A T E S ; i++)\{ \\
& \quad \text { if }\left(\left(\text { is_labeled }\left(g_{i}\right)=F A L S E\right) \&\left(\text { parity }\left(g_{i}\right)=\operatorname{parity}(g)\right)\right)\{ \\
& \quad \text { label_fanout }\left(g_{i}, T M P\right) \\
& \quad \text { compatible }=\text { dffs_check }\left(g_{i}, \text { parity }(g), \text { rule }\right) \\
& \quad \text { if }(\text { compatible })\{ \\
& \quad \text { label_fanout }\left(g_{i}, F O\right) \\
& \quad \mathcal{S}=\mathcal{S} \cup\left\{g_{i}\right\} \\
& \}
\end{aligned}
$$

$C O M P A T I B L E S$ starts by labeling "FO" the fanout cone of $g$, as no gates in that cone can belong to a compatible set containing $g$. Labeled gates represents elements of the set $F O(\mathcal{S})$. All gates $g_{i}$ that are not yet labeled and have the correct parity are then examined for insertion in $\mathcal{S}$. To this purpose, the fanout of $g_{i}$ that is not already in $F O(\mathcal{S})$ is temporarily labeled " $T M P$ ", and then visited by $d f s \_$seck in order to check the satisfaction of rule. If $g_{i}$ is compatible, it becomes part of $\mathcal{S}$ and its fanout is merged with $F O(\mathcal{S})$. The depth-first traversal of $d f s_{-}$check is stopped whenever the primary outputs or gates already in $F O(\mathcal{S})$ are reached, or a violation of rule is detected.

\section{References}

[1] R. Rudell and A. Sangiovanni-Vincentelli, "Multiple-valued minimization for PLA optimization," IEEE Transactions on CAD/CAS, vol. 6 , no. 5, pp. 727-750, Sept. 1987.

[2] E. L. Lawler, "An approach to multilevel boolean minimization," $A C M$ Joumal, vol. 11, no. 3, pp. 283-295, July 1964.

[3] R. K. Brayton, R. Rudell, A. Sangiovanni-Vincentelli, and A. R. Wang, "MIS: A multiple-level logic optimization system," IEEE Transactions on CAD/ICAS, vol. 6, no. 6, pp. 1062-1081, Nov. 1987.

[4] S. Muroga, Y. Kambayashi, H. C. Lai, and J. N. Culliney, "The transduction method - design of logic networks based on permissible functions," IEEE Transactions on Computers, vol. 38, no. 10, pp. 14041424 , Oct. 1989.

[5] K. A. Bartlett, R. K. Brayton, G. D. Hachtel, R. M. Jacoby, C. R. Morrison, R. L. Rudell, A. Sangiovanni-Vincentelli, and A. R. Wang, "Multilevel logic minimization using implicit don't cares," IEEE Transactions on CAD/CAS, vol. 7, no. 6, pp. 723-740, June 1988.

[6] R. Brayton and F. Somenzi, "An exact minimizer for boolean relations," in ICCAD, Proceedings of the International Conference on Computer-Aided Design, pp. 316-319, Nov. 1989.

[7] E. J. McCluskey, Logic Design Principles With Emphasis on Testable Semicustom Circuits. Prentice-Hall, 1986.

[8] S. W. Jeong and F. Somenzi, "A new algorithm for the binate covering problem and its application to the minimization of boolean relations," in ICCAD, Proceedings of the Intermational Conference on ComputerAided Design, pp. 417-420, 1992.

[9] M. Damiani, J. Yang, and G. De Micheli, "Optimization of combinational logic circuits based on compatible gates," tech. rep., Computer Systems Laboratory, Stanford University, 1993.

[10] R. E. Bryant, "Graph-based algorithms for boolean function manipulation," IEEE Transactions on Computers, vol. 35, no. 8, pp. 677-691, Aug. 1986.

[11] H. Savoj, R. K. Brayton, and H. Touat, "Extracting local don't cares and network optimization," in ICCAD, Proceedings of the Intemational Conference on Computer-Aided Design, pp. 514-517, Nov. 1991. 